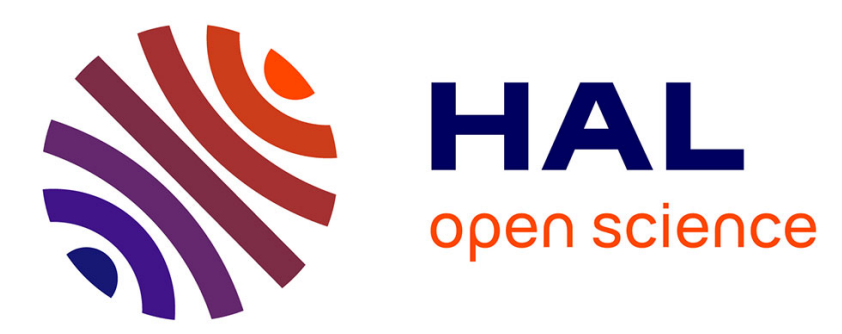

\title{
Seven-Parameter Linear Viscoelastic Model Applied to Acoustical Damping Materials
}

Emmanuel Gourdon, Cédric Sauzeat, Hervé Di Benedetto, Kévin Bilodeau

\section{To cite this version:}

Emmanuel Gourdon, Cédric Sauzeat, Hervé Di Benedetto, Kévin Bilodeau. Seven-Parameter Linear Viscoelastic Model Applied to Acoustical Damping Materials. Journal of Vibration and Acoustics Transactions of the ASME, 2015, 137 (6), pp.061003. 10.1115/1.4030719 . hal-01829841

\section{HAL Id: hal-01829841 \\ https://hal.science/hal-01829841}

Submitted on 27 Oct 2020

HAL is a multi-disciplinary open access archive for the deposit and dissemination of scientific research documents, whether they are published or not. The documents may come from teaching and research institutions in France or abroad, or from public or private research centers.
L'archive ouverte pluridisciplinaire HAL, est destinée au dépôt et à la diffusion de documents scientifiques de niveau recherche, publiés ou non, émanant des établissements d'enseignement et de recherche français ou étrangers, des laboratoires publics ou privés. 


\section{Seven-Parameter Linear Viscoelastic Model Applied to Acoustical Damping Materials}

In this paper, linear viscoelastic rheological properties of acoustical damping materials are predicted. A rheological model, based on a mechanical element approach, is pre-sented. It consists of a combination of two springs, two parabolic elements, and one dash-pot (2S2P1D). This model is applied to different acoustical damping materials. Its specificity comes from the fact that elements might be linked to structural and physical features. Parameters might be experimentally determined by tests. Application of the 2S2P1D linear viscoelastic model can adequately predict the behavior of acoustical damping materials with good accuracy. If the material verifies the time-temperature superposition principle (TTSP), the proposed model can predict the behavior on a wide frequency range, even with a small number of available data.
E. Gourdon ${ }^{1}$

Ecole Nationale des Travaux Publics de l'Etat, LGCB, LTDS UMR

CNRS5513, Rue Maurice Audin, Vaulx en Velin Cedex 69518, France

C. Sauzéat

Ecole Nationale des Travaux Publics de l'Etat, LGCB, LTDS UMR

CNRS5513, Rue Maurice Audin, Vaulx en Velin Cedex 69518, France

H. Di Benedetto

Ecole Nationale des Travaux Publics de l'Etat, LGCB, LTDS UMR

CNRS5513, Rue Maurice Audin, Vaulx en Velin Cedex 69518, France

K. Bilodeau

Bauval, 3550 Butte-aux-Renards, Varennes, QC J3X 1P7, Canada

\section{Introduction}

Nowadays, acoustical foams [1-4] and acoustical composite materials [5] are widely used for sound and vibration damping in building or automotive applications. To suppress vibrations, viscoelastic materials are usually placed on the surfaces of structures. To predict the responses of the damped structure and to design it, it is important to know the properties of the foams in the frequency range relevant to their application, so in a wide frequency range for acoustic applications.

Biot's theory [6,7] is used to describe the dynamic behavior of porous medium when the skeleton is set in motion. This theory uses the viscoelastic properties of the solid phase.

Moreover, it must be underlined that the viscoelastic properties of these acoustic foams are strongly frequency and temperature dependent as emphasized by lots of experimental works on the subject [8-14]. To describe the frequency and temperature dependence efficiently, computerized numerical methods require a mathematical model on the dynamic properties.

To experimentally determine those viscoelastic properties of acoustical foams, different techniques are used and explained in literature. They are mainly based on mass-spring resonance [11], standing wave resonance of a longitudinally excited rod with end mass [11], or based on nonresonant techniques [12,15]. An extension of certain measurements on a wide frequency range can be performed by using the TTSP as done by Ref. [16]. So, a theoretical modelization of the viscoelastic behavior of foams is needed to enhance the frequency range.

For all those reasons, a mathematical form of frequency dependences of dynamic properties is required. Modeling the viscoelastic behavior is an old problem not only in damping polymer materials but also in the field of bituminous materials

\footnotetext{
${ }^{1}$ Corresponding author.

${ }^{2}$ Former affiliation: Laboratoire Génie Civil et Bâtiment and LTDS UMR CNRS 5513, ENTPE, Rue Maurice Audin, 69518 Vaulx-en-Velin Cedex, France, an EIFFAGE Travaux Publics, R\&D Dept., 8 rue du Dauphinû CS 74005, 69964 Corbas, France.
}

[17-19]. Empirical models have been used [20,21], but the disadvantages of empirical models are that they are not related to the general constitutive equation of viscoelastic materials and it is difficult to understand the physics. Moreover, to apply a mathematical model to damping material, the model parameters should be easily estimated through experiments. In many existing models, those parameters are determined using curve-fitting procedures and are not necessarily linked to physical parameters, which could be estimated by other means (e.g., a static parameter).

Recently, a five-parameter fractional-derivative model [22] has been used to describe the dynamic behavior of viscoelastic materials such as polymeric damping material. The specificity of this model is due to the fact that the relevant constitutive equation contains time derivatives of stress and strain at different orders; so under certain conditions, this model is physically meaningful. The fractional-derivative model has become a powerful solution that describes the frequency-dependent dynamic characteristics of damping materials [23-25]. Even if the physical meaning of each parameter is defined, it is underlined in Ref. [22] that for some parameters "the precise meaning of [them] is not known yet" and we can only relate them to the high- or low-frequency behavior. Especially, at high frequencies, the modulus tends to infinity and not to a finite value. Then, complex algorithms based on nonlinear optimization and suboptimal methods may be used to estimate the parameters of foams modeled by a fractional-derivative model [26]. Kim and Lee [27] propose efficient identification method using different optimization techniques. Kim and Lee [23] propose an identification procedure using a finite element model of an unconstrained beam, a gradient-base numerical search algorithm, and a sensitivity analysis. Even if the algorithms can be very robust, efficient, and can reduce considerably the experiments, the physical meaning of parameters is lost, and it is difficult to link an optimized parameter to physical meaning.

Recently, the authors of Refs. [28-36] developed a rheological model based on a mechanical element approach for modeling the linear viscoelastic behavior of bitumens and asphalts, called 2S2P1D model. This model consists of a combination of two springs, two parabolic elements, and one dashpot. It seems useful to consider the simple behavior of analogical models constructed from mechanical elements to model linear viscoelastic behavior. 
Thanks to the 2S2P1D model, it is possible to obtain a physical model with physical parameters, which can be determined experimentally by other methods. The advantage of this approach is that the elements might be relatable to structural features. The 2S2P1D analogical model has a continuous spectrum, i.e., can be represented by an infinite number of Kelvin-Voigt elements in serie or Maxwell elements in parallel [37].

The aim of this paper is to show that the 2S2P1D linear viscoelastic model can be applied to acoustical damping materials with success. The objective of this paper is to show that a mathematical form with a physical meaning can be used to describe the linear viscoelastic behavior of acoustical foams with physical parameters.

This paper is divided into two parts. First, the 2S2P1D linear viscoelastic model is presented. The physical meaning of this model is described. The differences with the classical fractionalderivative models are shown. Second, the model is applied to three different materials. First, a polymeric damping material $[22,38]$ is considered. Even if this first damping material is not an acoustical material, it has been chosen here to validate the 2S2P1D model as done by Ref. [22] for the five-parameter fractional-derivative model. The same data have been used. The data are covering about ten decades. Second, the model is applied to an open-cell polyurethane foam [39] used as acoustic insulator. For this material, the complex shear modulus data were obtained by using a quasi-static method. In this case, the TTSP may be used. Then, the 2S2P1D model is applied to a polyvinyl chloridebased viscoelastic material where experimental data have been obtained by a modified Oberst beam method [40]. In this case, few experimental data are available (only the three or four first resonance peaks of the Oberst beam for each isotherm can be used). This last case is chosen to show the ability of the proposed model to predict the viscoelastic behavior of damping material even if few experimental data are available.

In these three cases, the 2S2P1D model shows its ability to predict the viscoelastic behavior on a wide frequency range with a good accuracy.

\section{2S2P1D Linear Viscoelastic Model}

Before describing the proposed 2S2P1D linear viscoelastic model, we present other viscoelastic models generally used in acoustical fields. This allows to see the differences introduced in the proposed model. This section is mainly based on a mechanical element approach, which is one of the originality of this paper since it has not been done previously in acoustical field.

Among the existing models for simulating linear viscoelastic behavior of acoustical damping materials, the four-parameter fractional Zener model has been found to be efficient to predict frequency variations with symmetrical loss factor peak. The mechanical representation of such a model is shown in Fig. 1.

The complex modulus is given by the following expression:

$$
G^{*}(j \omega \tau)=G_{00}+\frac{G_{0}-G_{00}}{1+(j \omega \tau)^{-\alpha}}
$$

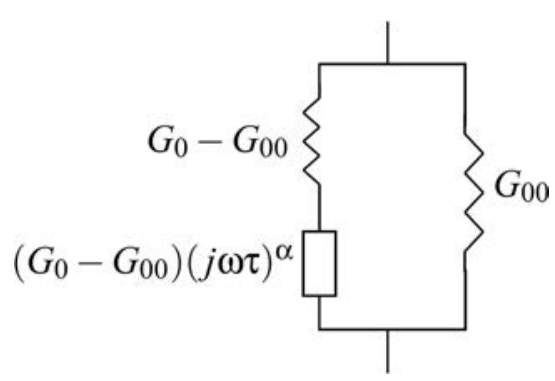

Fig. 1 Mechanical representation of the fractional Zener model where $\tau$ is the relaxation time, the parameters $G_{0}$ and $G_{00}$ have modulus dimension and $0<\alpha<1 . G_{00}$ is naturally the static modulus and $G_{0}$ is the glassy modulus.

It comes from the following constitutive equation:

$$
\sigma(t)+\tau^{\alpha} \frac{\mathrm{d}^{\alpha}}{\mathrm{d} t^{\alpha}} \sigma(t)=G_{00} \varepsilon(t)+G_{0} \tau^{\alpha} \frac{\mathrm{d}^{\alpha}}{\mathrm{d} t^{\alpha}} \varepsilon(t)
$$

where $\sigma(t)$ is the stress, $\varepsilon(t)$ is the strain, and $t$ is the time.

To take into account asymmetrical loss factor peak and the high-frequency behavior of polymeric damping materials, the authors of Ref. [22] have introduced a five-parameter fractional model. The complex modulus for the five-parameter model is given by the following expression:

$$
G^{*}(j \omega \tau)=G_{00}+\left(G_{\infty}-G_{00}\right) \frac{(j \omega \tau)^{\alpha}}{1+(j \omega \tau)^{\beta}}
$$

where $0<\beta<\alpha<1$.

It comes from the following constitutive equation:

$$
\begin{aligned}
\sigma(t)+\tau^{\beta} \frac{\mathrm{d}^{\beta}}{\mathrm{d} t^{\beta}} \sigma(t)= & G_{00} \varepsilon(t)+G_{\infty} \tau^{\beta} \frac{\mathrm{d}^{\beta}}{\mathrm{d} t^{\beta}} \varepsilon(t) \\
& +\left(G_{\infty}-G_{00}\right) \tau^{\alpha} \frac{\mathrm{d}^{\alpha}}{\mathrm{d} t^{\alpha}} \varepsilon(t)
\end{aligned}
$$

As underlined by the authors of Ref. [22], $G_{00}$ is naturally the static modulus and $G_{\infty}$ is related to the high-frequency behavior of the dynamic modulus, but "here, in contrast to the fourparameter model, $G_{\infty}$ is not the limit value (when $\omega \rightarrow \infty$ ) of the complex modulus" [22].

In the present paper, we want to have a mechanical element approach, which is why we decide to obtain the mechanical representation of this model as shown in Fig. 2.

With this representation, we can see that a spring is missing in series with the two parabolic elements to obtain a parameter, which will be a limit value of the modulus when $\omega \rightarrow \infty$.

Moreover, as underlined by Ref. [22], in relation to the highfrequency behavior, it should be noted that the five-parameter model is not intended to be used beyond a certain frequency, and this model is aimed to be used within a frequency range where the asymmetry of the loss peak and the peculiar high-frequency behavior of some polymeric materials can be observed.

To avoid mentioned drawbacks, other existing models can be used. In the field of pavement materials, bituminous mixes reveal to be viscoelastic materials if small strain and low number of cycles are applied. The authors of Refs. [28-30] have introduced a general model, which consists of a generalization of the Huet-Sayegh model [19]. This model is called 2S2P1D and is based on a simple combination of physical elements. This model is derived from the Huet-Sayegh model [19]. (For the Huet-Sayegh model, a second parabolic element is added in serie

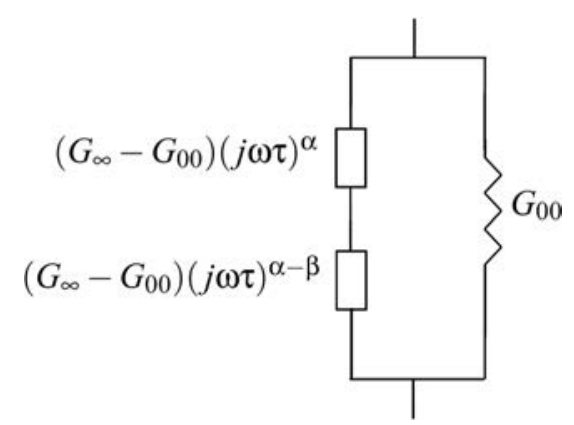

Fig. 2 Mechanical representation of the five-parameter fractional-derivative model 


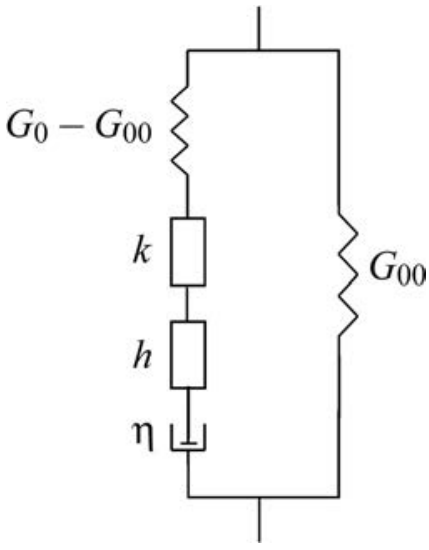

Fig. 3 Mechanical representation of the 2S2P1D model. $h$ and $k$ are the two parabolic creep elements.

in the branch where there is already a parabolic element in the Zener model.) The Huet-Sayegh model [19] has been adapted by adding a linear dashpot in serie with the two parabolic elements and the spring of rigidity $G_{0}-G_{00}$ (cf. Fig. 3).

Like the Huet-Sayegh model, the 2S2P1D model has a continuous spectrum (i.e., can be represented by an infinity of Kelvin-Voigt elements in serie or Maxwell elements in parallel).

At a given temperature, the introduced 2S2P1D model has seven constants and its complex modulus is given by the following expression:

$$
G^{*}(j \omega \tau)=G_{00}+\frac{G_{0}-G_{00}}{1+\delta(j \omega \tau)^{-k}+(j \omega \tau)^{-h}+(j \omega \beta \tau)^{-1}}
$$

with $j$ being the complex number defined by $j^{2}=-1, \omega$ is the pulsation $=2 \pi f$ ( $f$ is the frequency), $k$ and $h$ are the exponents such as $0<k<h<1, \delta$ is a constant, $G_{00}$ is the static modulus when $\omega \rightarrow 0, G_{0}$ is the glassy modulus when $\omega \rightarrow \infty$, and $\eta$ is the Newtonian viscosity (cf. Fig. 3). $\eta=\left(G_{0}-G_{00}\right) \beta \tau ; \beta$ is dimensionless.

$\tau$ is a characteristic time, which value varies only with temperature. It accounts for TTSP

$$
\tau(T)=a_{T}(T) \cdot \tau_{0}
$$

where $a_{T}(T)$ is the shift factor at temperature $T$ and $\tau_{0}=\tau\left(T_{\text {ref }}\right)$ is determined at the reference temperature $T_{\text {ref }}$.

It has to be emphasized that seven constants $\left(\delta, k, h, G_{00}, G_{0}, \beta\right.$, and $\left.\tau_{0}\right)$ are needed to entirely determine the linear viscoelastic behavior of the considered material, at a given temperature.

Furthermore, if the hypothesis of a linear viscoelastic thermorheologically simple behavior can be applied to the considered materials (which means that the TTSP holds), only the $\tau$ parameter depends on temperature.

If the TTSP holds, the shift factor at temperature $T, a_{T}(T)$ may be determined by means of different laws. For example, it can be determined by the Williams-Landel-Ferry empirical expression [41] or by using the approximated Kramers-Kronig relations [42]. However, parameters of these laws are often determined by curvefitting [43] in order to best superimpose the isotherms and they can have poor precision. In the present paper, we have determined it along with the parameters of the viscoelastic model (as it is done by some authors $[44,45])$. As underlined in Ref. [46], the calculated shift coefficients with this last method have a physical meaning since the viscoelastic model is supposed to verify some conditions such as causality [47]. It should be underlined that Ref. [46] calculated the shift coefficients by a method requiring fulfillment of the Kramers-Kronig relations conveying the causality condition.

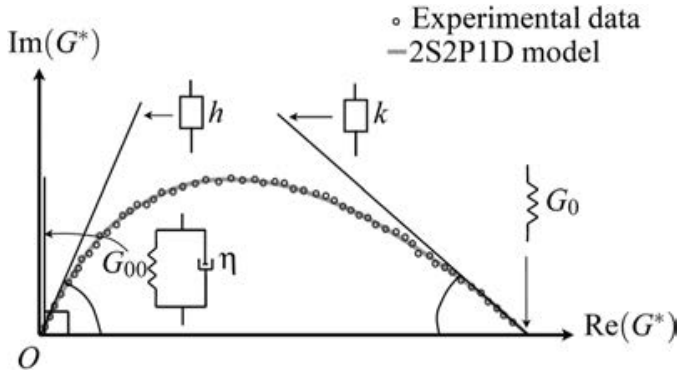

Fig. 4 Complex modulus in Cole-Cole axes

Like the fractional model, it is possible to obtain a constitutive equation for the 2S2P1D model

$$
\begin{aligned}
\sigma(t) & +b_{1} \frac{\mathrm{d}}{\mathrm{d} t} \sigma(t)+b_{2} \frac{\mathrm{d}^{\alpha_{1}}}{\mathrm{~d} t^{\alpha_{1}}} \sigma(t)+b_{3} \frac{\mathrm{d}^{\alpha_{2}}}{\mathrm{~d} t^{\alpha_{2}}} \sigma(t) \\
= & a_{0} \varepsilon(t)+a_{1} \frac{\mathrm{d}}{\mathrm{d} t} \varepsilon(t)+a_{2} \frac{\mathrm{d}^{\alpha_{1}}}{\mathrm{~d} t^{\alpha_{1}}} \varepsilon(t)+a_{3} \frac{\mathrm{d}^{\alpha_{2}}}{\mathrm{~d} t^{\alpha_{2}}} \varepsilon(t)
\end{aligned}
$$

where $\quad \alpha_{1}=1-h, \alpha_{2}=1-k \quad\left(0<\alpha_{1}<\alpha_{2}<1\right) . \quad b_{1}=\beta \tau$. $b_{2}=\delta \beta \tau^{\alpha_{1}}, b_{3}=\beta \tau^{\alpha_{2}}, a_{0}=G_{00}, a_{1}=G_{0} b_{1}, a_{2}=G_{00} b_{2}, a_{3}=G_{00} b_{3}$.

This is the first time that the constitutive equation of the 2S2P1D model has been written. It allows to better understand the interest of the proposed model. Indeed, the coefficient $b_{1}$ of the first derivative of $\sigma$ allows to take into account the viscosity and the coefficient $a_{1}$ of the first derivative of $\varepsilon$ allows to take into account the viscosity and the glassy modulus when $\omega \rightarrow \infty$.

Those two first derivatives were not present in the two previous models (fractional Zener and five-parameter fractional models).

At low frequencies, the five-parameter fractional-derivative model is equivalent to a parabolic element, whereas the 2S2P1D model is equivalent to a dashpot element (and a spring in parallel). Moreover, one spring is added in serie to the two parabolic elements to obtain a physical meaning for high frequencies: the modulus tends to a finite value $G_{0}$ at high frequencies. Thanks to the 2S2P1D model, it is possible to obtain a physical model with physical parameters which can be determined experimentally by other methods (for example, by using ultrasonic waves for the determination of a parameter at high frequencies as performed in Refs. [48-50]) and which have a precise physical meaning as shown in Fig. 4. The advantage of this approach is that the elements might be relatable to structural features.

From the results on acoustical damping materials, we will show that this comprehensive model translates correctly the linear viscoelastic behavior in the small strain domain for any range of frequencies and temperatures.

We can identify the parameters by the following procedure.

If limit values are known by experimental tests or by identification from asymptotic values and from the variation of the complex modulus (it is possible to read off the model parameters directly from the experimental data because the frequency curves are smooth as illustrated in Fig. 4), they constitute the initial values of the parameters to be optimized by the algorithm.

If experimental data do not cover a frequency range large enough to observe the asymptotic behavior, we find initial parameters estimates that are closed to the optimal values. A good way to do this is by simulations. Both the measured and calculated curves are compared and the parameters are adjusted by hand until the agreement between the two is reasonably good. Although this will be a subjective judgment, it is sufficient to find a good starting point for the algorithm refinement. Using those parameters improve robustness of the algorithm. We use a more stable algorithm than the Gauss-Newton algorithm allowing to find a solution even if the initial values are far from the optimal values.

The parameters are then optimized and refined by the following procedure. We minimize the cost function $F_{\text {cost }}$, which is defined 
(a)

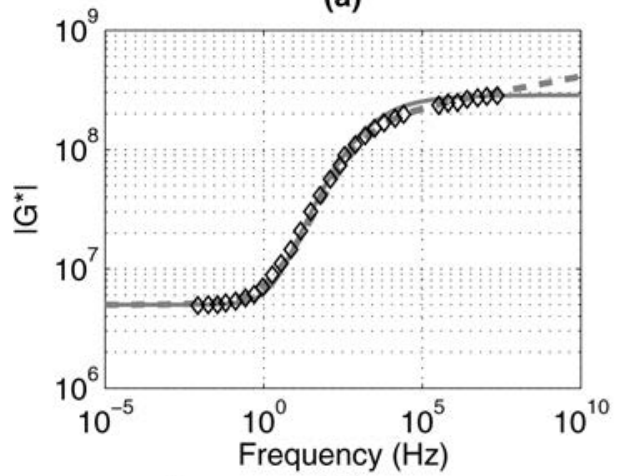

(c)

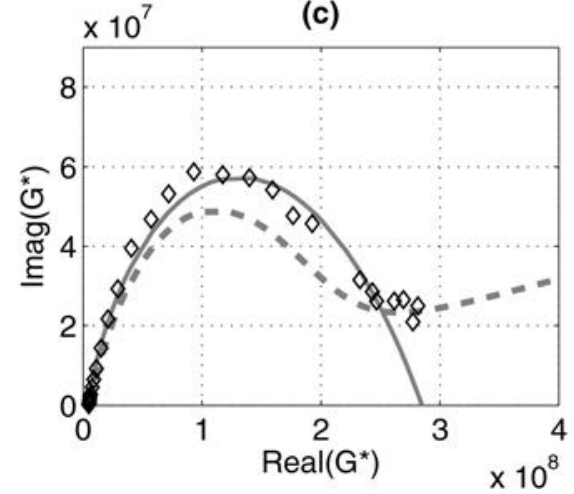

(b)

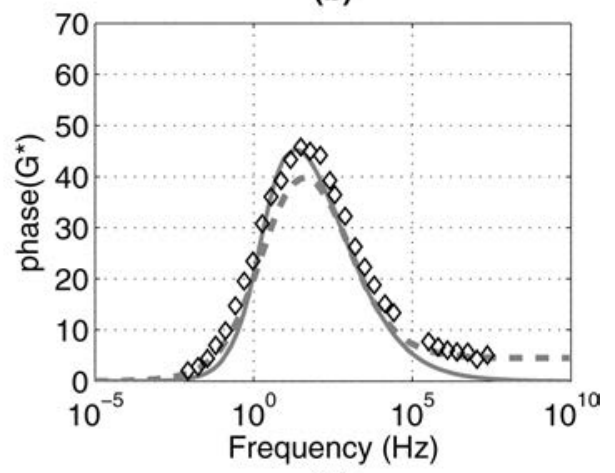

(d)

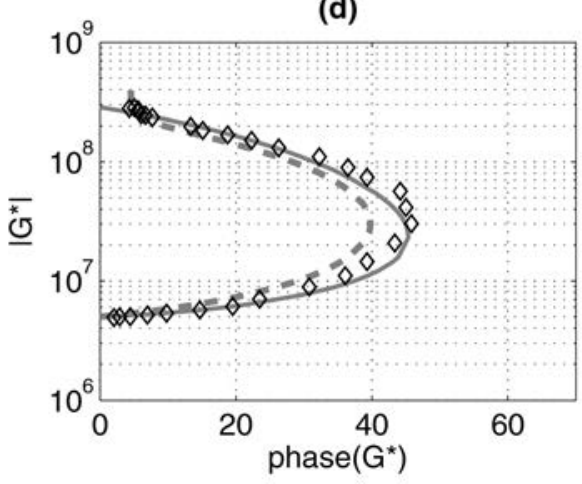

Fig. 5 Cyclic shear properties of a polymeric damping material: (a) master curve of norm of $G^{*}$ (in Pa); (b) master curve of phase angle of $G^{\star}$ (in degree); (c) complex modulus (in Pa) in Cole-Cole axes; and (d) complex modulus (in $\mathrm{Pa}$ ) in black space. The gray continuous line represents the 2S2P1D model, the gray dashed line represents the five-parameter fractionalderivative model, and the diamonds represent experimental measurements.

by (with $G_{\text {meas }}$ being the quantity measured and $G_{\text {model }}$ being the quantity output of the model)

$F_{\text {cost }}=\sum_{f} \frac{\left|\operatorname{Re}\left(G_{\text {model }}\right)-\operatorname{Re}\left(G_{\text {meas }}\right)\right|^{2}}{\left|\operatorname{Re}\left(G_{\text {meas }}\right)\right|^{2}}+\frac{\left|\operatorname{Im}\left(G_{\text {model }}\right)-\operatorname{Im}\left(G_{\text {meas }}\right)\right|^{2}}{\left|\operatorname{Im}\left(G_{\text {meas }}\right)\right|^{2}}$

To obtain the minimum of this function, we can use classical least-squares curve fitting algorithms. For example, we can use the Levenberg-Marquardt method [51-53]. This method uses a search direction that is a cross between the Gauss-Newton direction and the steepest descent direction. This local optimization algorithm demonstrates great efficiency provided that initial values enable to avoid being trapped in local minima and valleys. So, the idea here is to find initial parameters (by comparisons between calculations and experimental values) such that the initial volatility is similar to the target volatility. Thus, a satisfying solution (either a global minimum or a good enough local minimum, which is in the error range of measures) may be located in the search space of the local optimization algorithm. This point has been underlined in Ref. [54] for this kind of algorithm.

For each case, we have verified that taking different "acceptable" initial values gives the same solution to be sure of the local convergence.

\section{Modeling of Acoustical Damping Materials}

Three different materials are considered.

First, a polymeric damping material $[22,38]$ is analyzed. Even if this first damping material is not an acoustical material, it has been chosen here to validate the 2S2P1D model as done by Ref. [22] for the five-parameter fractional-derivative model. The same data have been used. When the author of Ref. [22] introduced his model, the first verification was done on this polymeric damping material. That is why we decide to use the same material. The data are covering about ten decades and the reliability of the experimental data will allow to validate the tested model on a wide frequency range.

Second, the model is applied to acoustical damping materials. The aim here is to show that the 2S2P1D model can be applied to acoustical damping materials with success. In the mean time, we want to show that the 2S2P1D model can also predict the viscoelastic behavior of acoustical foams even if few experimental data are available, which is an originality of this paper. Thus, the model is first applied to an open-cell polyurethane foam [39] used as acoustical isolator. For this material, the complex shear modulus data were obtained by using a quasi-static method and the TTSP has to be used to predict the viscoelastic behavior on a wide frequency range. Then, the $2 \mathrm{~S} 2 \mathrm{P} 1 \mathrm{D}$ model is applied to a polyvinyl chloride-based viscoelastic material where experimental data (only the three or four first resonance peaks of the Oberst beam can be used for each temperature) have been obtained by a modified Oberst beam method [40]. In this case, few experimental data are available, and we will show that it is possible to predict the viscoelastic behavior on a large frequency range.

3.1 Case of a Polymeric Damping Material. A polymeric damping material $[22,38]$ is considered. The experimental values of the cyclic shear properties of the commercial available damping material are taken from Ref. [38] and are plotted in Fig. 5. The cyclic properties were measured by the composite beam method at several temperatures. As underlined in Ref. [22], "data covering about ten decades of frequency were determined by means of the frequency-temperature equivalence principle." The 
Table 1 Parameter values of the polymeric damping material determined for the 2S2P1D model

\begin{tabular}{ccccccc}
\hline$G_{00}(\mathrm{~Pa})$ & $G_{0}(\mathrm{~Pa})$ & $k$ & $h$ & $\delta$ & $\beta$ & $\tau$ \\
\hline $5 \times 10^{6}$ & $2.85 \times 10^{8}$ & 0.44 & 0.86 & 2.5 & 200 & $8 \times 10^{-4}$ \\
\hline
\end{tabular}

low scatter and the smoothness of the reduced frequency curves testify the reliability of the experimental data.

The 2S2P1D model is applied to this material. The initial parameters can be easily determined directly from the experimental data (for example by taking the limit values) and optimized then by the procedure described in Sec. 2. For this material, the model parameters are given in Table 1.

As shown in Fig. 5, there is a good agreement between the 2S2P1D model and the experimental data over a wide frequency range.

The author of Ref. [22] has already compare his model (five parameters: $G_{00}=5 \times 10^{6} \mathrm{~Pa}, G_{\infty}=1.8 \times 10^{8} \mathrm{~Pa}, \alpha=0.605$, $\beta=0.554, \tau=2.09 \times 10^{-4}$ ) to these data. The cost function $F_{\text {cost }}$ (presented in the previous function) for the five parameters model is approximately the double of the optimized cost function for the 2S2P1D model (even if this cost function is small for the two models). It should be noticed that this difference can also be explained by the fact that the optimization process is not the same in Ref. [22] and in the present paper. In the present paper, we have optimized $F_{\text {cost }}$ whereas a curve fitting on $\operatorname{Re}\left(G^{*}\right), \operatorname{Imag}\left(G^{*}\right)$ and on the loss factor was performed in Ref. [22]. In the two cases, the mean of the relative error between model and experimental results is less than $10 \%$. The 2S2P1D model shows superior performance to the fiveparameter fractional-derivative model, but the number of parameters is seven. It should be noticed that additional two coefficients (one term in fractional derivative) in fractional-derivative model may also show better performance.
Table 2 Parameter values of the open-cell polyurethane foam determined for the 2S2P1D model $\left(T_{\text {ref }}=+10^{\circ} \mathrm{C}\right)$

\begin{tabular}{lcccccc}
\hline$G_{00}(\mathrm{~Pa})$ & $G_{0}(\mathrm{~Pa})$ & $k$ & $h$ & $\delta$ & $\beta$ & $\tau$ \\
\hline $1.65 \times 10^{4}$ & $2.09 \times 10^{6}$ & 0.215 & 0.22 & 79 & 2 & 0.0374 \\
\hline
\end{tabular}

3.2 Case of a Polyurethane Foam. Now, the model can be applied to an open-cell polyurethane foam used as acoustic isolator. The experimental data are taken from Ref. [39]. In Ref. [39], four materials were studied. These materials have the same chemical composition, the same porosity, and the same mass density. They only differ by their pore sizes. So here, we just consider one of these foams (all the results for the other foams are similar because the polymer, which composes skeleton, is identical for these foams). The considered foam is named as Bulpren S20 foam. For this material, the complex shear modulus data were obtained by using a quasi-static method on a small frequency range and the TTSP has to be used to predict the viscoelastic behavior on a wide frequency range.

In Ref. [39], measurements were repeated at various temperatures above the glass transition of the polyurethane $\left(T_{g}\right.$ $=-25^{\circ} \mathrm{C}$ ). In the present paper, e.g., we use six isotherms: from $-21^{\circ} \mathrm{C}$ to $+10{ }^{\circ} \mathrm{C}$. Experimental data are plotted in Fig. 6 .

A reference temperature can be arbitrarily chosen. Then if the TTSP can be applied to this material, the model can predict $G^{*}$ or phase angle of $G^{*}$ to frequencies which are not accessible experimentally up to $10^{8} \mathrm{~Hz}$. In Ref. [39], the authors show that the TTSP can be applied to this material. So, for this material, the shift factor $a_{T}(T)$ must also be determined (along with the parameters of the viscoelastic model). The reference temperature $T_{\text {ref }}=+10^{\circ} \mathrm{C}$ is arbitrarily chosen. For this material, the 2S2P1D model parameters are given in Table 2. Figure 7 shows that the 2S2P1D model is well adapted to predict the linear viscoelastic behavior of this foam. Thanks to the TTSP, it is possible to obtain
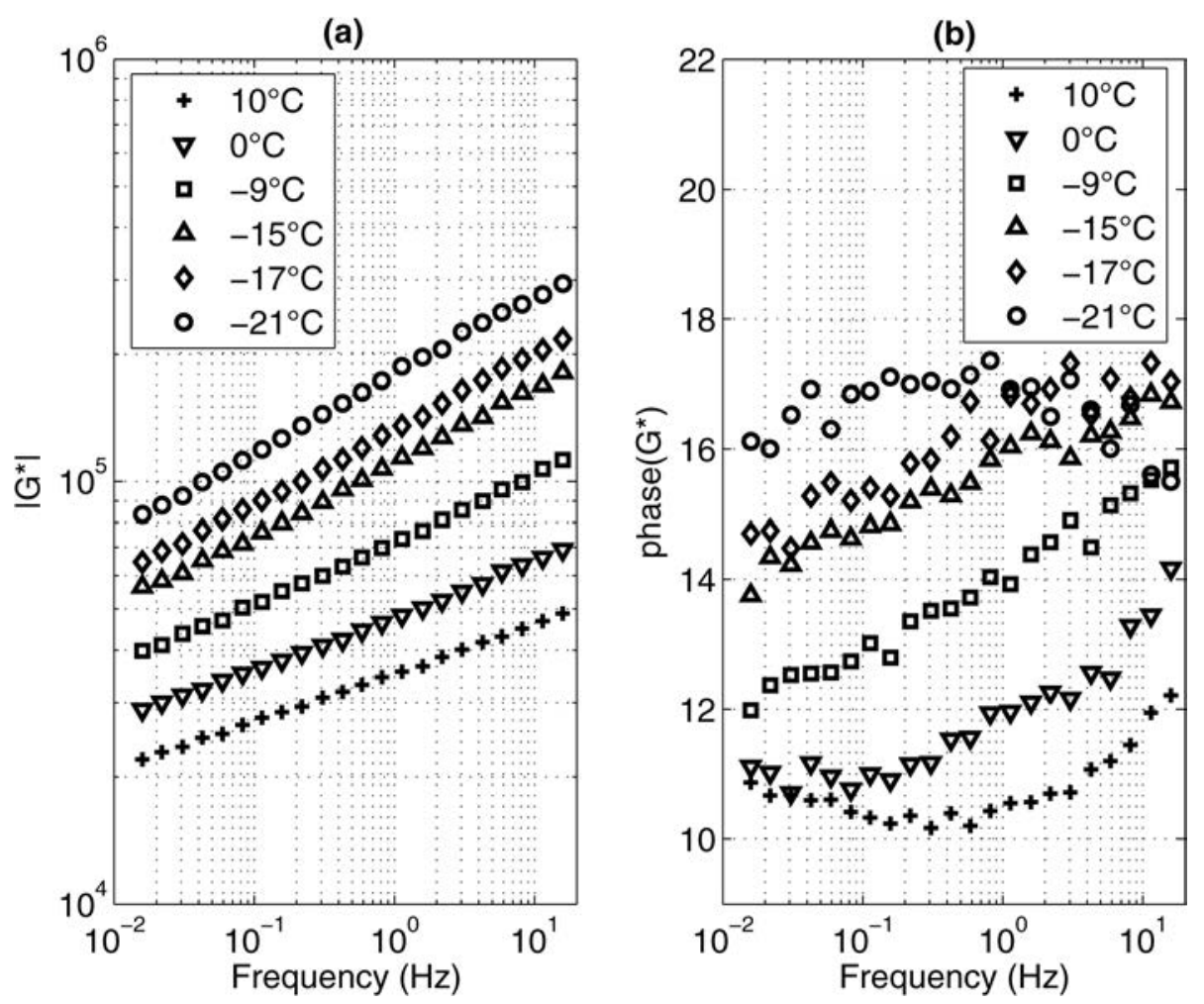

Fig. 6 Cyclic shear properties of a polyurethane foam: (a) norm of $G^{\star}$ (in $\mathrm{Pa}$ ) for six isotherms and $(b)$ phase angle (in degree) of $G^{\star}$ for six isotherms 
(a)

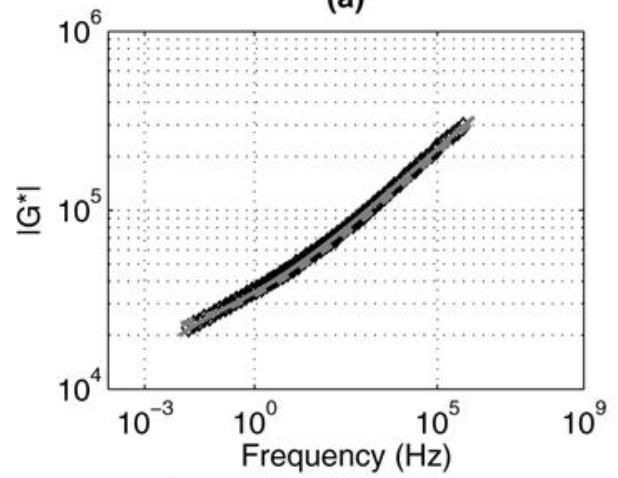

(c)

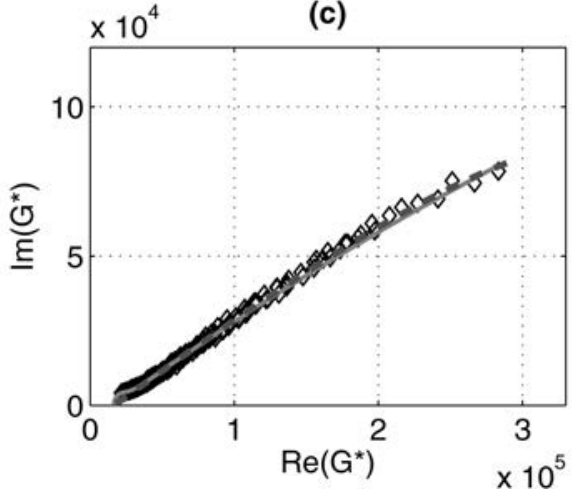

(b)

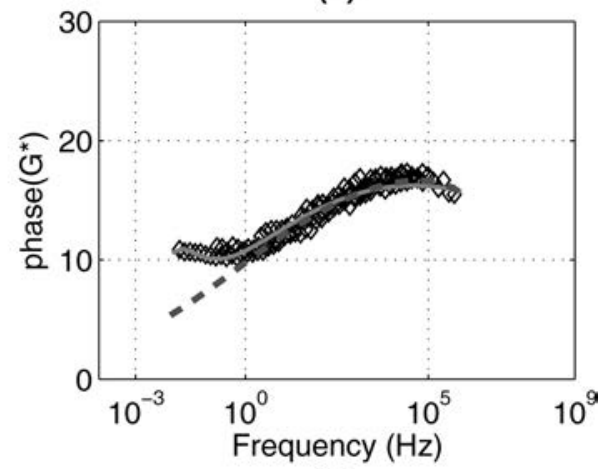

(d)

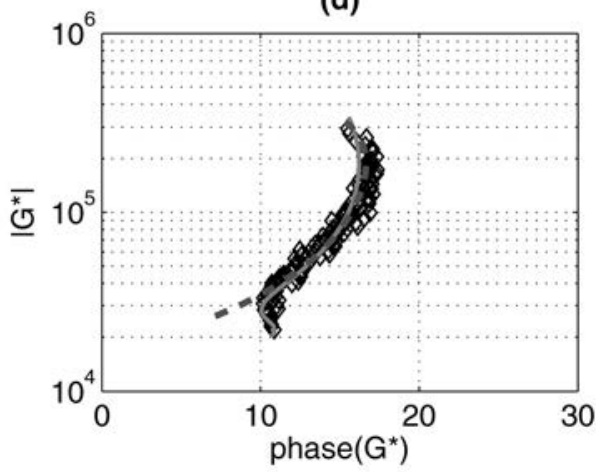

Fig. 7 Cyclic shear properties of a polyurethane foam: (a) master curve of norm of $G^{\star}$ (in $\mathrm{Pa}$ ); (b) master curve of phase angle of $\mathrm{G}^{*}$ (in degree); (c) complex modulus (in $\mathrm{Pa}$ ) in Cole-Cole axes; and (d) complex modulus (in $\mathrm{Pa}$ ) in black space. The gray continuous line represents the 2S2P1D model, the gray dashed line represents the five-parameter fractionalderivative model, and the diamonds represent experimental measurements $\left(T_{\text {ref }}=+10^{\circ} \mathrm{C}\right)$.

the master-curve on a wide frequency range. This effect is well predicted by the 2S2P1D model.

Even with a lower variation of phase angle of $G^{*}$, it should be noticed that the 2S2P1D model predicts the small variation of the phase of $G^{*}$ with good accuracy. For this material, we can also apply the five-parameter fractional-derivative model. After optimization, the parameters of this model are: $G_{00}=1.65 \times 10^{4} \mathrm{~Pa}$, $G_{\infty}=9.9 \times 10^{5} \mathrm{~Pa}, \alpha=0.21, \beta=0.5$, and $\tau=8 \times 10^{-10}$.

The accuracy of the two models is good, as shown in Fig. 7 where the simulations of the two models have been shown. For example, the mean relative error between experimental results and modelization of the norm of the shear modulus is $1.2 \%$ for the 2S2P1D model and $4.9 \%$ for the five-parameter fractionalderivative model.

Nevertheless, it should be underlined that, even if the accuracy is good, the five-parameter fractional-derivative model cannot predict the slight increase of the phase at low frequencies, as shown in Fig. 7. In the black space $(d)$, we clearly see that it is impossible to have an inflection of the curve at low frequencies with only five parameters in the model. The experimental measurements clearly show that when the modulus of $G^{*}$ is decreasing at low frequencies, the phase is slightly increasing. This behavior can only be modelized by using the 2S2P1D model, which has more parameters. Indeed, the behavior of this foam, at low frequencies, is influenced by the viscous response of the polymer which composes the skeleton of this foam. The presence of the dashpot (to take into account the Newtonian viscosity $\eta$ ) is essential at low frequencies to describe this kind of behavior. Without this dashpot (like in the five-parameter fractional-derivative model), the behavior at low frequencies is equivalent to a parabolic element and it is impossible to modelize the slight increase of phase at low frequencies.

So, we have shown that the 2S2P1D model can be applied to acoustical damping materials with success. In the mean time, we want to show that the 2S2P1D model can also predict the viscoelastic behavior of acoustical foams even if few experimental data are available, that is why we consider another material.

3.3 Case of a Polyvinyl Chloride-Based Viscoelastic Material. The 2S2P1D model has been applied to a polyvinyl chloride-based viscoelastic material where experimental data (only the three or four first resonance peaks of the Oberst beam can be used for each temperature) have been obtained by a modified Oberst beam method [40]. This modified Oberst beam method is an inexpensive and simple method to quickly determine the Young's modulus of viscoelastic materials. However, for a given temperature, only the three or four first resonance modes of the beam can be used, this explains why we only get three points for each isotherm. However, the 2S2P1D model can be a good mean to predict the behavior between those points. Even if small number of experimental data is available, this example shows that it is possible to predict the behavior on a wide frequency range. So the modified Oberst beam method becomes a powerful and simple mean to experimentally determine the viscoelastic behavior of acoustical damping materials.

With the modified Oberst beam method, measurements were repeated for six temperatures: from $+5^{\circ} \mathrm{C}$ to $+30^{\circ} \mathrm{C}$. Experimental data are plotted in Fig. 8.

Then, to see if the TTSP holds, we just plot the measurements data in the Cole-Cole axes (Fig. 9(c)). In the Cole-Cole axes, the measurements data form a single continuous master curve so the TTSP holds. Thus, for this material, the shift factor $a_{T}(T)$ must also be determined (along with the parameters of the viscoelastic model). The reference temperature $T_{\text {ref }}=+5^{\circ} \mathrm{C}$ is arbitrarily chosen.

For this material, the 2S2P1D model parameters are given in Table 3. 
(a)

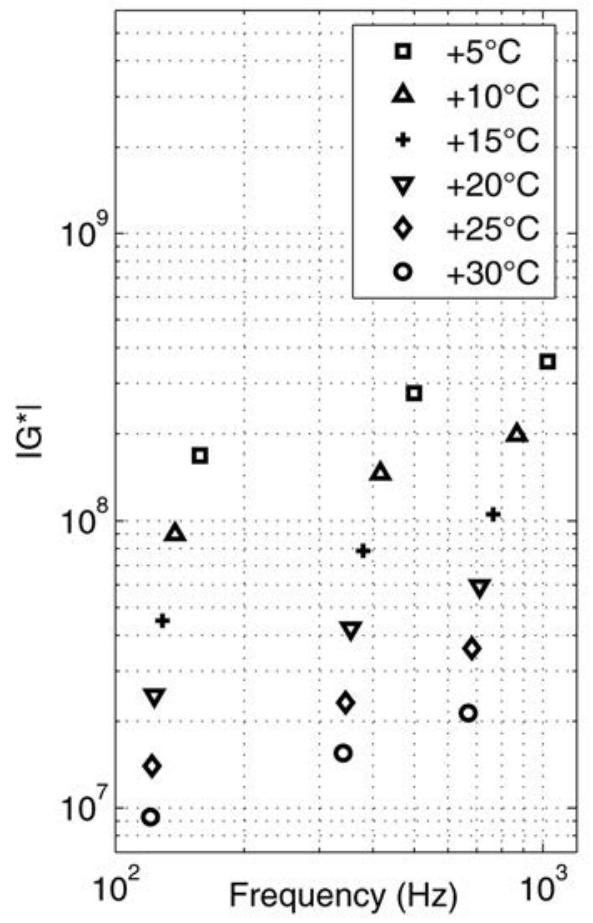

(b)

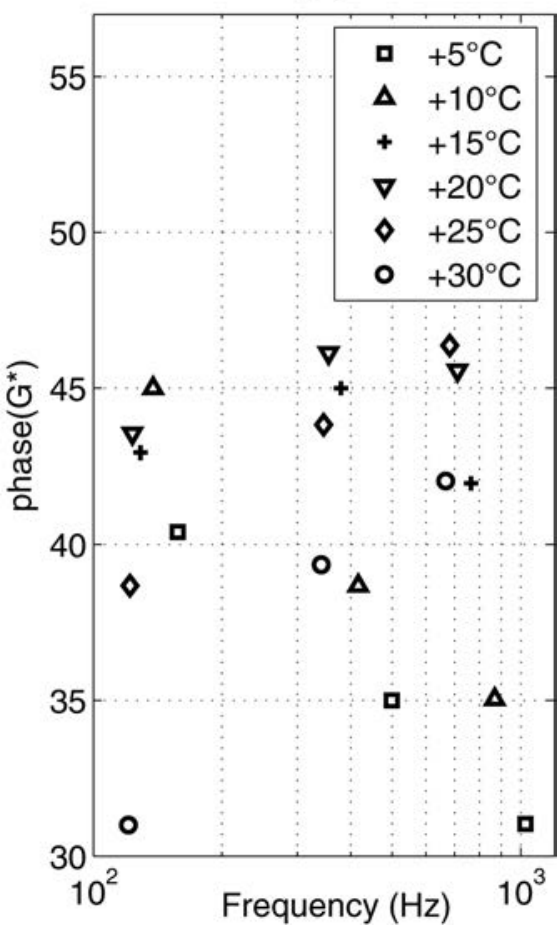

Fig. 8 Young's modulus properties of a polyvinyl chloride-based viscoelastic material: (a) norm of $E^{\star}$ (in $\mathrm{Pa}$ ) for six isotherms and $(b)$ phase angle (in degree) of $E^{\star}$ for six isotherms

(a)

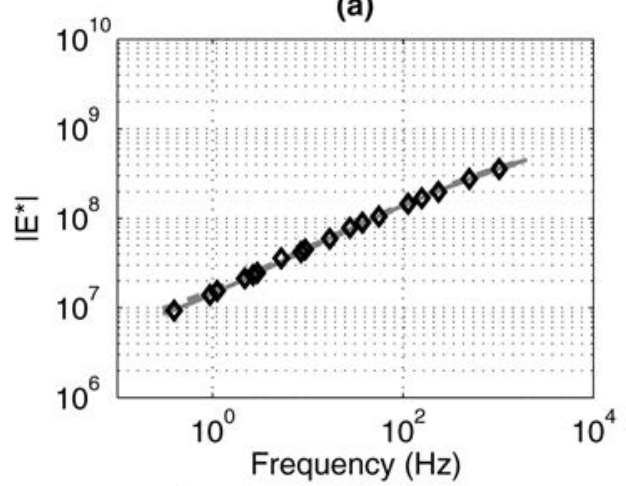

(c)

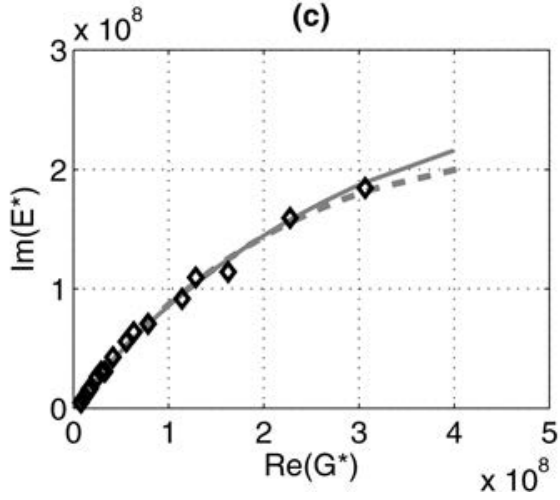

(b)

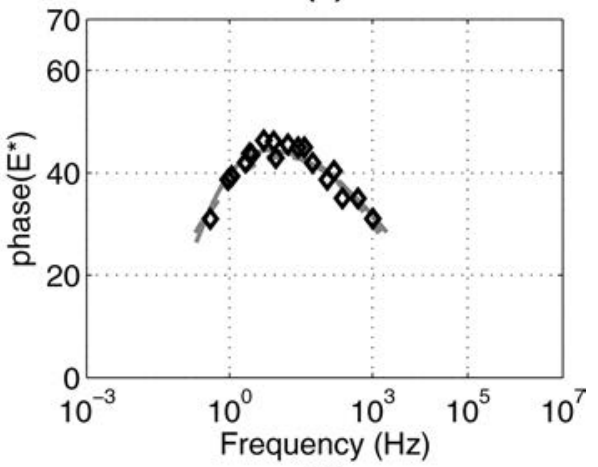

(d)

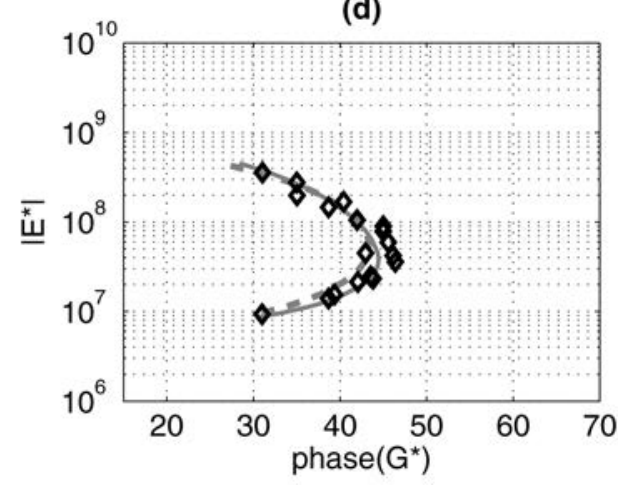

Fig. 9 Young's modulus properties of a polyvinyl chloride-based viscoelastic material: (a) master curve of norm of $E^{*}$ (in $\mathrm{Pa}$ ); (b) master curve of phase angle of $E^{*}$ (in degree); (c) complex modulus (in $\mathrm{Pa}$ ) in Cole-Cole axes; and $(d)$ complex modulus (in $\mathrm{Pa}$ ) in black space. The gray continuous line represents the 2S2P1D model, the gray dashed line represents the five-parameter fractional-derivative model, and the diamonds represent experimental measurements $\left(T_{\text {ref }}=+5^{\circ} \mathrm{C}\right)$. 
Table 3 Parameter values of the polyvinyl chloride-based viscoelastic material determined for the 2S2P1D model $\left(T_{\text {ref }}=+5^{\circ} \mathrm{C}\right)$

\begin{tabular}{ccccccc}
\hline$E_{00}(\mathrm{~Pa})$ & $E_{0}(\mathrm{~Pa})$ & $k$ & $h$ & $\delta$ & $\beta$ & $\tau_{0}$ \\
\hline $5 \times 10^{6}$ & $1.24 \times 10^{9}$ & 0.45 & 0.77 & 5.6 & 16 & $9.8 \times 10^{-4}$ \\
\hline
\end{tabular}

Figure 9 shows that by using few experimental data (only the three resonance peaks of the Oberst beam can be used), the 2S2P1D model can supply physical information about the viscoelastic behavior since it can predict the viscoelastic behavior with a good precision. The low accuracy of phase of $G^{*}$ measurements (which is deduced by structural damping coefficient measurements) can explain the small differences between the theoretical model and experimental values even if the global accuracy is good.

For this material, we can also apply the five-parameter fractional-derivative model. After optimization of the two models, the mean relative error between experimental results and modelization of the norm of the Young modulus is $1.7 \%$ for the 2S2P1D model and $3.7 \%$ for the five-parameter fractional-derivative model.

\section{Conclusions}

A rheological model called 2S2P1D based on a mechanical element approach and developed for bituminous materials has been applied to model the linear viscoelastic behavior of different acoustical damping materials. It consists of a combination of two springs, two parabolic elements, and one dashpot. This model is physically meaningful. In the case of the polymeric damping material, the data are covering about ten decades and the reliability of the experimental data allows to validate the tested model on a wide frequency range.

The case of the polyurethane foam shows that the 2S2P1D model can be used with the TTSP to predict the viscoelastic behavior of open-cell foam on a wide frequency range. The presence of the dashpot (to take into account the Newtonian viscosity $\eta$ ) is essential at low frequencies to describe the slight increase of phase. Without this dashpot (like in the five-parameter fractionalderivative model), the behavior at low frequencies is equivalent to a parabolic element and it is impossible to modelize this slight increase.

The last case shows that by using few experimental data (only the three or four first resonance peaks of the modified Oberst beam [40] can be used), the 2S2P1D model can supply physical information about the viscoelastic behavior since it can predict the viscoelastic behavior with a good accuracy.

To conclude, 2S2P1D linear viscoelastic model can predict the behavior of acoustical damping materials with good accuracy on a wide frequency range thanks to the TTSP even if small number of experimental data are available.

It remains to study more precisely the variations of the physical parameters of the model as a function of the parameters of the foam such as the pore-size or the density.

\section{Acknowledgment}

This work was performed within the framework of the LABEX CELYA (No. ANR-10-LABX-0060) of Université de Lyon, within the program "Investissements d'Avenir" (No. ANR-11IDEX-0007) operated by the French National Research Agency (ANR).

\section{References}

[1] Zwikker, C., and Kosten, C., 1949, Sound Absorbing Materials, Elsevier, Amsterdam.
[2] Allard, J., 1993, Propagation of Sound in Porous Media: Modelling Sound Absorbing Materials, Elsevier, Essex, UK.

[3] Olny, X., Sgard, F., Perrot, C., and Panneton, R., 2004, "Microscopic and Mesoscopic Approaches for Describing and Building Sound Absorbing Porous Materials," Proceedings of the Second TUL-ENTPE Workshop, Szklarska Poreba, Poland, Mar. 3-6, pp. 187-206.

[4] Lind-Nordgren, E., and Gransson, P., 2010, "Optimising Open Porous Foam for Acoustical and Vibrational Performance," J. Sound Vib., 329(7), pp. 753-767.

[5] Gourdon, E., and Seppi, M., 2010, "Extension of Double Porosity Model to Porous Materials Containing Specific Porous Inclusions," Acta Acust. Acust., 96(2), pp. 275-291.

[6] Biot, M., 1956, "The Theory of Propagation of Elastic Waves in a Fluidsatured Porous Solid. I. Low Frequency Range,” J. Acoust. Soc. Am., 28(2), pp. $168-191$.

[7] Biot, M., 1956, "The Theory of Propagation of Elastic Waves in a Fluidsatured Porous Solid. II. Higher Frequency Range,” J. Acoust. Soc. Am., 28(2), pp. 168-191.

[8] Capps, R., 1983, "Dynamic Young's Moduli of Some Commercially Available Polyurethanes," J. Acoust. Soc. Am., 73(6), pp. 2000-2005.

[9] Oyadiji, S., and Tomlimson, G., 1985, "Determination of the Complex Moduli of Viscoelastic Structural Elements by Resonance and Non-Resonance Techniques," J. Sound Vib., 101(3), pp. 277-298.

[10] Corsaro, R., and Sperling, L., 1990, Sound and Vibration Damping With Polymers, American Chemical Society, Washington, DC.

[11] Pritz, T., 1994, "Dynamic Young's Modulus and Loss Factor of Plastic Foams for Impact Sound Isolation," J. Sound Vib., 178(3), pp. 315-322.

[12] Mariez, E., Sahraoui, S., and Allard, J., 1996, "Elastic Constants of Polyurethane Foams Skeleton for Biot Model," 25th International Congress on Noise Control Engineering (Inter-Noise 96), Liverpool, UK, July 30-Aug. 2, pp. 951-954.

[13] Dauchez, N., Etchessahar, M., and Sahraoui, S., 2002, "On Measurements of Mechanical Properties of Sound Absorbing Materials," Second Biot Conference on Poromechanics, Grenoble, France, Aug. 26-28, pp. 627-632.

[14] Pritz, T., 1990, "Non-Linearity of Frame Dynamic Characteristics of Mineral and Glass Wool Materials," J. Sound Vib., 136(2), pp. 263-274.

[15] Panneton, R., and Langlois, C., 2001, "Polynomial Relation for the Mechanical Characterization of Poroelastic Materials," 17th International Congress of Acoustics (ICA '01), Rome, Italy, Sept. 2-7, Paper No. 4A.08.06.

[16] Etchessahar, M., Sahraoui, S., Benyahia, L., and Tassin, J., 2005, "Frequency Dependence of Elastic Properties of Acoustic Foams," J. Acoust. Soc. Am., 117(1), pp. 1114-1121.

[17] Van der Poel, C., 1954, "A General System Describing the Viscoelastic Properties of Bitumens and Its Relation to Routinr Test Data," J. Appl. Chem., 4(5), pp. 231-236.

[18] Heukolem, W., and Klomp, J., 1964, "Road Design and Dynamic Loading," Proceedings of the Association of Asphalt Paving Technologists, Ann Arbor, MI, Vol. 33, pp. 92-125.

[19] Sayegh, G., 1967, "Viscoelastic Properties of Bituminous Mixtures," Second International Conference on Structural Design of Asphalt Pavement, Ann Arbor, MI, pp. 743-755.

[20] Havriliak, S., and Negami, S., 1967, "A Complex Plane Representation of Dielectric and Mechanical Relaxation Processes in Some Polymers," Polymer, 8, pp. 161-210.

[21] Hartmann, B., Lee, G. F., and Lee, J., 1994, "Loss Factor Height and Width Limits for Polymer Relaxations," J. Acoust. Soc. Am., 95(1), pp. 226-233.

[22] Pritz, T., 2003, "Five-Parameter Fractional Derivative Model for Polymeric Damping Materials," J. Sound Vib., 265(5), pp. 935-952.

[23] Kim, S.-Y., and Lee, D.-H., 2009, "Identification of Fractional-DerivativeModel Parameters of Viscoelastic Materials From Measured Frfs," J. Sound Vib., 324(3-5), pp. 570-586.

[24] ASTM, 2010, "Standard Test Method for Measuring Vibration-Damping Properties of Materials," ASTM International, West Conshohocken, PA, ASTM Standard E756-05(2010)

[25] Jones, D., 2001, Handbook of Viscoelastic Vibration Damping, Wiley, New York.

[26] Deng, R., Davies, P., and Bajaj, A., 2003, "Flexible Polyurethane Foam Modeling and Identification of Viscoelastic Parameters for Automotive Seating Applications," J. Sound Vib., 262(3), pp. 391-417.

[27] Kim, S.-Y., and Lee, D.-H., 2009, "Identification of Fractional-DerivativeModel Parameters of Viscoelastic Materials From Measured FRFs," J. Sound Vib., 324(3-5), pp. 570-586.

[28] Olard, F., and Di Benedetto, H., 2003, “General '2S2P1D' Model and Relation Between the Linear Viscoelastic Behaviours of Bituminous Binders and Mixes," Road Mater. Pavement Des., 4(2), pp. 185-224.

[29] Olard, F., Di Benedetto, H., and Vaniscote, J.-C., 2005, "Properties of Bituminous Mixtures at Low Temperatures and Relations With Binder Characteristics," Mater. Struct., 38(1), pp. 121-126.

[30] Di Benedetto, H., Olard, F., Sauzéat, C., and Delaporte, B., 2004, "Linear Viscoelastic Behaviour of Bituminous Materials: From Binders to Mixes," Road Mater. Pavement Des., 5(Suppl. 1), pp. 163-202.

[31] Di Benedetto, H., Neifar, M., Sauzéat, C., and Olard, F., 2007, "ThreeDimensional Thermo-Viscoplastic Behaviour of Bituminous Materials: The DBN Model," Road Mater. Pavement Des., 8(2), pp. 285-316.

[32] Yin, H., Buttlar, W., Paulino, G., and Di Benedetto, H., 2008, "Assessment of Existing Micro-Mechanical Models for Asphalt Mastics Considering Visco- 
elastic Effects," Road Mater. Pavement Des., 9(1), pp. 31-57.

[33] Delaporte, B., Di Benedetto, H., Chaverot, P., and Gauthier, G., 2009, "Linea Viscoelastic Properties of Bituminous Materials Including New Products Made With Ultrafine Particles," Road Mater. Pavement Des., 10(1), pp. 7-38.

[34] Izzi, Y. M. N., Mounier, D., Ginoux, M., Mohd, H., Airey, G., and Di Benedetto, H., 2013, "Modelling the Rheological Properties of Bituminous Binders Using the 2S2P1D Model," Constr. Build. Mater., 38, pp. 395-406.

[35] Mangiafico, S., Di Benedetto, H., Sauzéat, C., Olard, F., Pouget, S., and Planque, L., 2014, "New Method to Obtain Viscoelastic Properties of Bituminen Blends From Pure and Rap Binder Constituents," Road Mater. Pavement Des., 15(2), pp. 312-329.

[36] Tapsoba, N., Sauzéat, C., Di Benedetto, H., Baaj, H., and Ech, M., 2014, "Behavior of Asphalt Mixtures Containing Rap and Shingles," Road Mater. Pavement Des., 15(2), pp. 330-347.

[37] Tiouajni, S., Di Benedetto, H., Sauzéat, C., and Pouget, S., 2011, “Approximation of Linear Viscoelastic Model by Generalized Kelvin Voigt or Generalized Maxwell Models: Application to Bituminous Materials in the 3 Dimensional Case," Road Mater. Pavement Des., 12(4), pp. 897-719.

[38] Fowler, B., 1989, "Interactive Characterization and Data Base Storage of Complex Modulus Data," Proceedings of Damping '89, West Palm Beach, FL, Feb. 8-10, Vol. 2, Paper No. FAA.

[39] Deverge, M., Benyahia, L., and Sahraoui, S., 2009, "Experimental Investigation on Pore Size Effect on the Linear Viscoelastic Properties of Acoustic Foams," J. Acoust. Soc. Am., 126(3), pp. 93-96.

[40] Wojtowicki, J.-L., Jaouen, L., and Panneton, R., 2004, "New Approach for the Measurement of Damping Properties of Materials Using the Oberst Beam," Rev. Sci. Instrum., 75(8), pp. 2569-2574.

[41] Williams, M., Landel, R., and Ferry, J., 1955, "The Temperature Dependence of Relaxation Mechanisms in Amorphous Polymers and Other Glassforming Liquids," J. Am. Chem. Soc., 77(14), pp. 3701-3707.

[42] Booij, H., and Thoone, G., 1982, "Generalization of Kramerskronig Transform and Some Approximations of Relations Between Viscoelastic Quantities," Rheol. Acta, 21(1), pp. 15-24.
[43] Gergesova, M., Zupancic, B., Saprunov, I., and Emri, I., 2011, "The Closed Form t-T-P Shifting (CFS) Algorithm," J. Rheol., 55(1), pp. 1-16.

[44] Madigosky, W., Lee, G., and Niemiec, J., 2006, "A Method for Modeling Polymer Viscoelastic Data and the Temperature Shift Function," J. Acoust. Soc. Am., 119(6), pp. 3760-3765.

[45] Guedes, R., 2011, "A Viscoelastic Model for a Biomedical Ultra-High Molecular Weight Polyethylene Using the Time-Temperature Superposition Principle," Polym. Test., 30(3), pp. 294-302.

[46] Rouleau, L., Deu, J.-F., Legay, A., and Lay, F. L., 2013, "Application of Kramers-Kronig Relations to Time-Temperature Superposition for Viscoelastic Materials," Mech. Mater., 65, pp. 66-75.

[47] Pritz, T., 1999, "Verification of Local Kramers-Kronig Relations for Complex Modulus by Means of Fractional Derivative Model," J. Sound Vib., 228(5), pp. 1145-1165.

[48] Di Benedetto, H., Sauzéat, C., and Sohm, J., 2009, "Stiffness of Bituminous Mixtures Using Ultrasonic Waves Propagation," Road Mater. Pavement Des., 10(4), pp. 789-814.

[49] Mounier, D., Di Benedetto, H., and Sauzéat, C., 2012, "Determination of Bituminous Mixtures Linear Properties Using Ultrasonic Wave Propagation," Constr. Build. Mater., 36, pp. 638-647.

[50] Gudmarsson, A., Ryden, N., Di Benedetto, H., Sauzéat, C., Tapsoba, N., and Birgisson, B., 2014, "Comparing Linear Viscoelastic Properties of Asphalt Concrete Measured by Laboratory Seismic and Tension-Compression Tests," J. Nondestr. Eval., 33(4), pp. 571-582.

[51] Levenberg, K., 1944, "A Method for the Solution of Certain Problems in LeastSquares,”Q. Appl. Math., 2, pp. 164-168.

[52] Marquardt, D., 1963, "An Algorithm for Least-Squares Estimation of Nonlinear Parameters," SIAM J. Appl. Math., 11(2), pp. 431-441.

[53] Moré, J., 1977, "The Levenberg-Marquardt Algorithm: Implementation and Theory," Numerical Analysis (Lecture Notes in Mathematics, Vol. 630), G. A. Watson, ed., Springer-Verlag, Heidelberg, Germany, pp. 105-116.

[54] Gauthier, P., and Rivaille, P.-Y. H., 2009, "Fitting the Smile, Smart Parameters for SABR and Heston," Social Science Research Network, Rochester, NY. 